\title{
OSCILLATORY MODES OF ENERGY TRANSPORT IN SOLAR MAGNETIC REGIONS*
}

\author{
R. E. Danielson and B.D. SAVAGE \\ (Princeton University Observatory, Princeton, N.J., U.S.A.)
}

\begin{abstract}
A B S T RACT
It appears that it is necessary to look for oscillatory (i.e. overstable) instabilities as the cause of energy transport in sunspots. Making use of recent calculations on the hydromagnetic stability of thermally unstable layers with open boundary conditions, it is found that oscillatory modes can occur in the interior of sunspots. Some possible consequences of these oscillatory modes are discussed.
\end{abstract}

\section{Introduction}

A long-standing problem in solar physics is the energy balance in a center of activity. This problem is often referred to as the missing energy in a sunspot, i.e.: what has happened to the energy the Sun would have radiated if the sunspot had not been present? This problem is still unsolved and will probably remain so until the mechanism of energy transport in sunspots is understood. For this reason, this paper will largely be concerned with the method of energy transport in a sunspot.

It is often tacitly assumed that some form of convection takes place in sunspots and that the convection motions are the main mechanism of energy transport. Some arguments to support this idea are as follows. First, some fine structure such as the small umbral dots reported by Danielson (1964) and Beckers and Schröter (1967) and the larger umbral granules reported by Bray and Loughhead (1959) suggests the presence of convection. Second, the 'turbulent velocities' observed in sunspots are comparable with those observed in the undisturbed photosphere (Howard, 1958; Elste, 1963; Elsässer and Fricke, 1965; Brückner, 1965) and suggest that some type of convective or oscillatory motion takes place. And thirdly, it is very difficult (if not impossible) to produce a self-consistent model of a sunspot with only radiative transport (Chitre, 1963).

However, hydromagnetic stability calculations have failed to reveal any unstable convective modes in the sunspot if we assume the presence of a vertical magnetic field of about 2500 gauss. In general this is to be expected because $H_{\text {eq }}$, the equipartition magnetic field (i.e. the magnetic field for which the magnetic-energy density equals the convective-energy density in the normal convective zone), is generally smaller than the magnetic field in the sunspot.

\footnotetext{
* Presented by R. E. Danielson.
} 
As shown in the appendix,

$$
H_{\mathrm{eq}} \leqslant 6600 \text { gauss }\left(\rho\left[\frac{\mathrm{gm}}{\mathrm{cm}^{3}}\right]\right)^{1 / 6}\left(\begin{array}{l}
l \\
h
\end{array}\right)^{1 / 3} .
$$

It is evident from Equation (1) that $H_{\mathrm{eq}}$ is quite insensitive to the value of the mixing length which one chooses. The value of $H_{\text {eq }}$ computed from Equation (1) is shown in Figure 1 as a function of depth in the normal convection zone. The values of $\rho$ which were used were taken from a table adapted by Böhm (1963) from a model (based on $l / h=1)$ computed by Böhm-Vitense (1958).

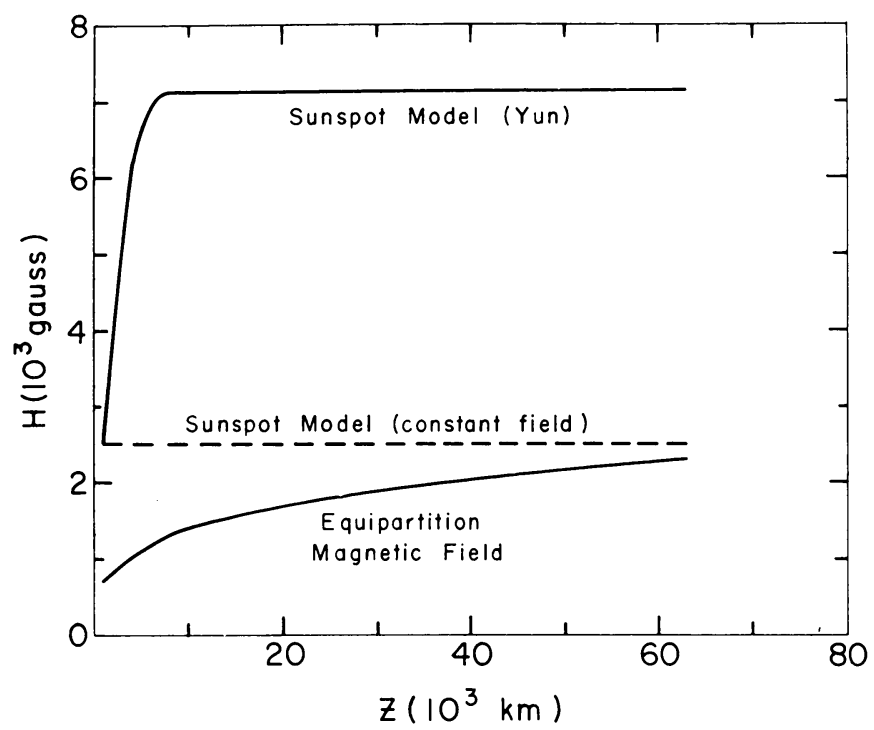

FIG. 1. The equipartition magnetic field and the magnetic field in a sunspot model of Yun (1967) as a function of depth (z) into the sunspot. Also shown is a constant field (2500 gauss) sunspot model.

It is clear from Figure 1 that the equipartition magnetic field is several times smaller than the field in a sunspot model computed by Yun (1967) following the methods of Deinzer (1965). Indeed for $l / h=1, H_{\text {eq }}$ does not exceed 2500 gauss (the surface field in Yun's model) for any depth in the convection zone. However, if $l / h=2, H_{\text {eq }}$ exceeds 2500 gauss at depths in excess of $30000 \mathrm{~km}$, but never exceeds the magnetic fields in Yun's model. The values of $H_{\text {eq }}$ calculated by Marik (1966) based on an earlier model of the convection zone (Vitense, 1953) are somewhat larger than given in Figure 1, but they do not exceed the magnetic fields in Yun's model.

Thus, with the possible exception of some very special modes, one would in general not expect any convection modes in sunspots. No such special modes have been found and therefore it is necessary to look for oscillatory (i.e. overstable) instabilities as the cause of energy transport in sunspots. Section 2 of this paper summarizes our present 
understanding of these oscillatory instabilities, while some possible consequences of them in sunspots are discussed in Section 3.

\section{Summary of Hydromagnetic Stability Calculations in the Presence of a Vertical Magnetic Field}

Early studies of hydromagnetic stabilities in the presence of a vertical magnetic field (Danielson, 1961; Weiss, 1964; for a general summary see Chandrasekhar, 1961) assumed a superadiabatic plane-parallel layer of depth $d$ and employed the Boussinesq approximation (Spiegel and Veronis, 1960). The free-free boundary conditions were used because they led to a very simple eigenfunction for $w$, the vertical (or $z$ ) component of the perturbation velocity, i.e.

$$
w=\left[\cos k_{x} x \cos k_{y} y \sin \begin{array}{r}
\pi z \\
\end{array}\right] e^{n t}
$$

where $k_{x}$ and $k_{y}$ are the horizontal wave numbers and where $n$ is the complex growth rate. We will call such eigenfunctions 'closed', and the corresponding boundary conditions (in this case the free-free boundary conditions) will be referred to as

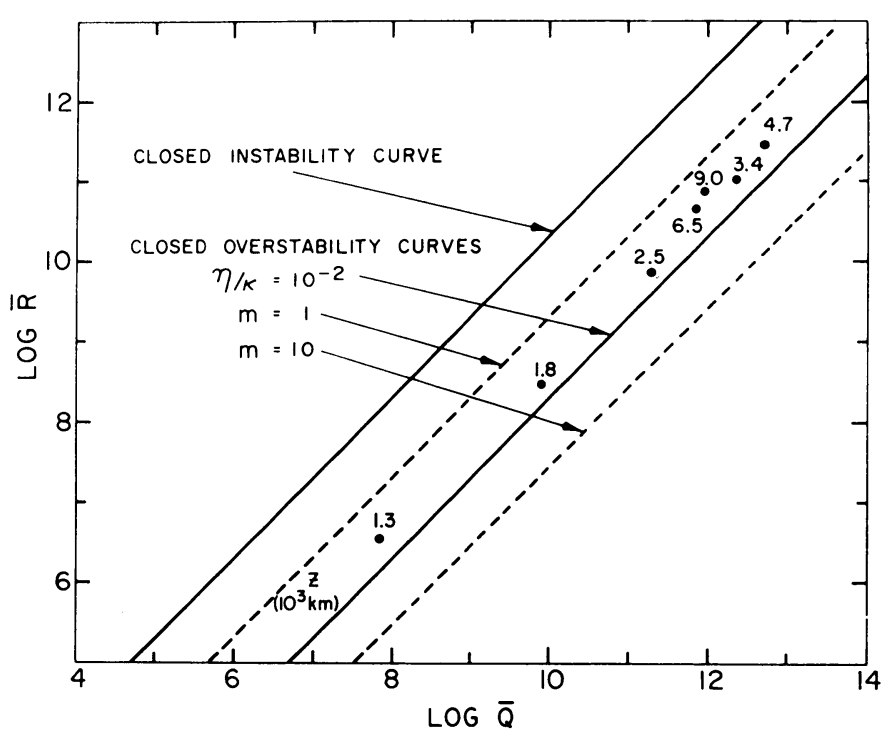

FIG. 2. An $R, \bar{Q}$ stability diagram for a closed fluid layer (no outgoing hydromagnetic waves permitted). Shown are closed instability and overstability curves. The overstability curve marked $\eta / \kappa=10^{-2}$ includes only resistive heating as a dissipation mechanism. The curves marked $m=1$ and 10 include the compressive heating mechanism of Kato (1966) as the means of dissipation. The points are the $R, \bar{Q}$ coordinates of Yun's (1967) sunspot model at each pressure scale height into the model. The depth (Z) into the model at which these quantities were computed is indicated. 
'closed' boundary conditions since they prohibit hydromagnetic wave emission from the superadiabatic layer.

The results of the calculations with the free-free boundary conditions are summarized in Figure 2, where the curves separate regions in the $\bar{R}, \bar{Q}$ plane according to the character of $n$. The dimensionless numbers $\bar{R}$ and $\bar{Q}$ are defined by:

$$
\bar{R}=\frac{g \alpha \beta d^{4}}{\kappa^{2} \pi^{4}} \quad \text { and } \quad \bar{Q}=\frac{\mu H^{2} d^{2}}{4 \pi^{3} \rho \kappa^{2}},
$$

where $g$ is the acceleration of gravity, $\alpha \approx 1 / T$ is the volume coefficient of expansion, $T$ is the temperature, $d$ is the depth of the superadiabatic layer, $\kappa$ is the radiative diffusivity, $\mu$ is the magnetic permeability, $H$ is the strength of the vertical magnetic field, $\rho$ is the mean density in the layer, and $\beta$ is the superadiabatic gradient defined by:

$$
\beta=-\left(\begin{array}{l}
\mathrm{d} T \\
\mathrm{~d} z
\end{array}+\begin{array}{l}
g \\
c_{P}
\end{array}\right) .
$$

In Equation (4), $\left(-g / c_{P}\right)$ is the adiabatic temperature gradient. The calculations assumed that the cell shape factor,

$$
s=\left(k_{x}^{2}+k_{y}^{2}\right)_{\pi}^{1 / 2} d
$$

was equal to unity and that $\eta / \kappa=10^{-2}$, where $\eta$ is the magnetic diffusivity. This value of $\eta / \kappa$ is approximately the mean value between $Z=2000$ and $Z=9000 \mathrm{~km}$ in Yun's (1967) sunspot model (see Figure 3). The exchange of stabilities curve is not shown because of its minor physical significance (Danielson, 1961).

The $\bar{R}, \bar{Q}$ coordinates of Yun's sunspot model at each pressure scale height are also shown in Figure 2. That is, the pressure has increased by a factor of $e$ at the depth represented by each point and the characteristic depth $d$ has been set equal to the local pressure scale height at each point. It is evident that the points lie about a factor of 30 below the closed instability curve. Approximately the same situation occurs for a uniform 2500-gauss field superimposed on the normal convection zone supporting the general conclusion (based on the equipartition magnetic field) that there are no unstable convective modes in the sunspot umbra. However, the points do lie somewhat above the overstability curve for joule heating, based on $\eta / \kappa=10^{-2}$, and therefore the possibility of overstable oscillations is not excluded. The overstability curve for compressive heating (Kato, 1966) is also shown for $m=V_{\mathrm{S}} / V_{\mathrm{A}}=1$ and $m=10$, where $V_{\mathrm{S}}$ and $V_{\mathrm{A}}$ are the sound and Alfvén velocities, respectively. The overstability curve for $m=4$ coincides with the overstability curve for $\eta / \kappa=10^{-2}$. However, Kato's simplified dispersion relation overestimates the dissipation for $m>1$ and therefore the true curves lie below the indicated ones. Since $m$ is greater than 10 for $Z$ greater than $7000 \mathrm{~km}$ (see Figure 4), compressive heating is probably less important than joule heating as a source of dissipation in the bulk of the sunspot interior. 


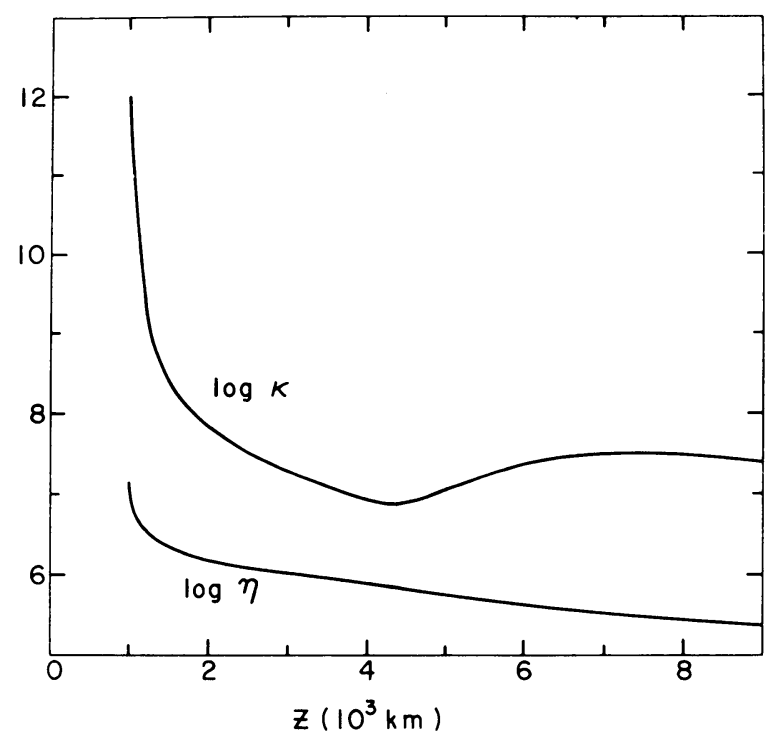

FIG. 3. Log $\kappa$ (the radiative diffusivity) and $\log \eta$ (the magnetic diffusivity) as a function of depth in Yun's (1967) sunspot model.

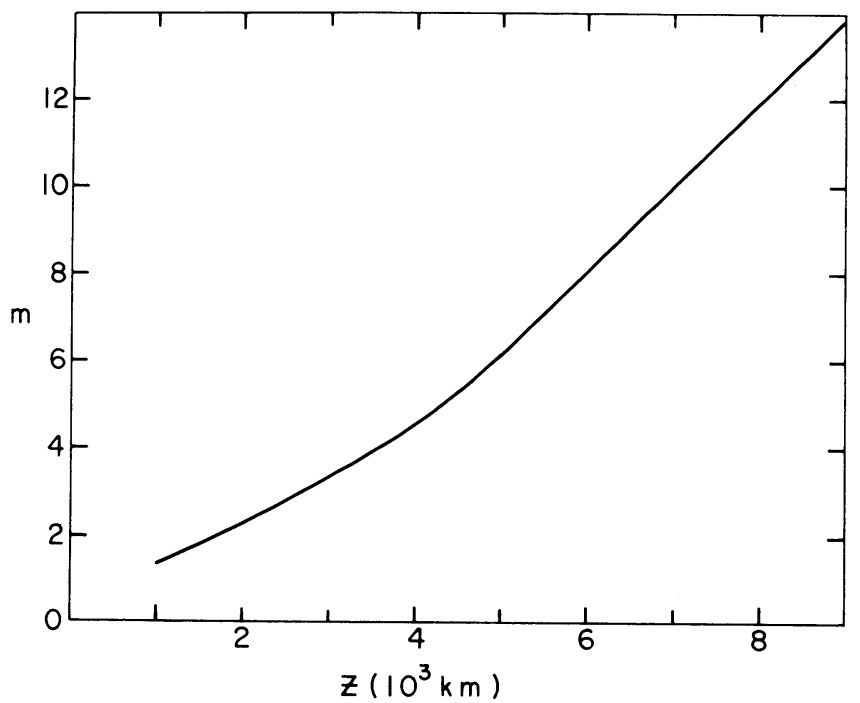

FIG. 4. $m=V_{\mathrm{S}} / V_{\mathrm{A}}$ as a function of depth in Yun's (1967) sunspot model.

However, the closed boudary conditions upon which Figure 2 is based are probably rather unrealistic because they do not allow any energy to be emitted from the layer in the form of hydromagnetic waves. The first calculations with open boundary conditions (Danielson, 1966; Musman, 1967) are shown in Figure 5. Again in the 
Boussinesq approximation, free-free boundary conditions were chosen for the bottom of the superadiabatic layer while the boundary conditions at the top of the layer were chosen in such a way as to allow hydromagnetic waves to propagate upwards into a semi-infinite adiabatic region.

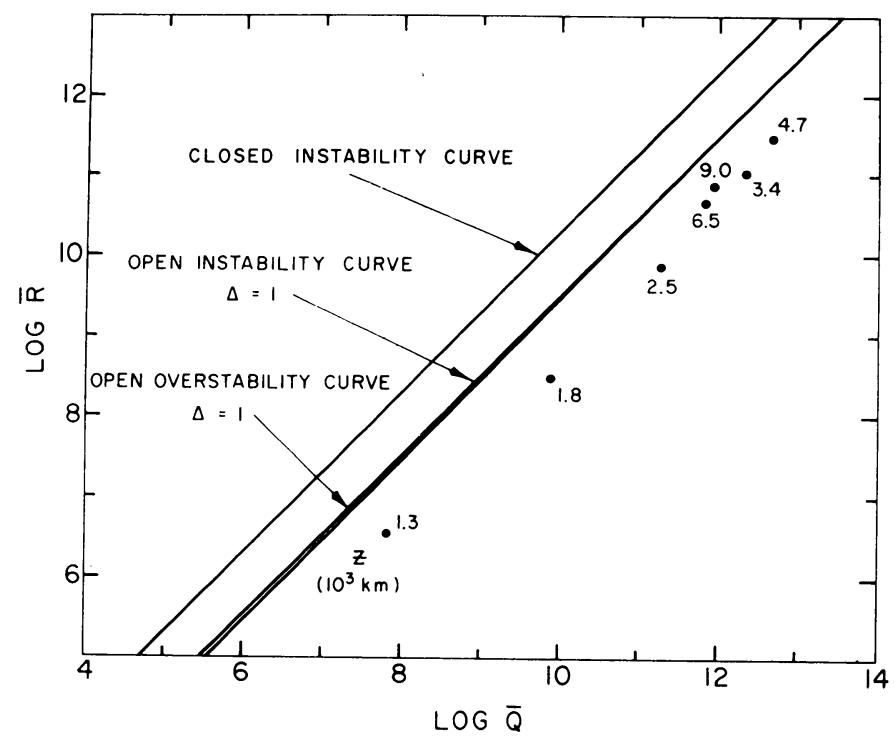

FIG. 5. $R, Q$ stability diagram for an open fluid layer (upward-propagating hydromagnetic waves are permitted). Shown are the open instability and overstability curves obtained by Musman (1967) for the case of no density discontinuity $\left(\Delta=\rho_{\mathrm{down}} / \rho_{\mathrm{up}}=1\right)$. The $R, \bar{Q}$ coordinates of Yun's (1967) sunspot model are also shown.

The open instability curve in Figure 5 is lower than the closed instability curve due to the fact that the open eigenfunction in the superadiabatic layer is approximately sinusoidal with a wavelength equal to $4 d$ while the closed eigenfunction has a wavelength of $2 d$. The largest change takes place in the open overstability curve because the damping caused by hydromagnetic wave emission is so large that the open overstability curve lies nearly as high in the $\bar{R}, \bar{Q}$ plane as the open instability curve. Furthermore, attempts to reduce the wave emission from the superadiabatic layer by introducing a discontinuity in the hydromagnetic velocity (by means of a density discontinuity between the superadiabatic and adiabatic regions) did not significantly affect the results (Danielson, 1965). The reason for this is that very large density discontinuities are required before a large fraction of the hydromagnetic waves are reflected.

Figure 5 repeats the $\bar{R}, \bar{Q}$ coordinates of Yun's model and it is evident that they lie below the open overstability curve. Thus on the basis of these calculations, one would 
conclude that the sunspot umbra is stable not only to convective overturning but also to overstable oscillations.

However, a density discontinuity introduces the possibility of surface gravity waves. The effect of these gravity waves was not included in Musman's (1967) calculations, but Savage (1967) has extended the calculations to include the presence of standing gravity waves at the interface. His results are summarized in Figure 6 where a new set of coordinates, $\hat{R}$ and $\hat{Q}$, are used. These coordinates are defined by

$$
\hat{R}=\begin{aligned}
& \bar{R} \\
& G
\end{aligned} \text { and } \hat{Q}=\begin{aligned}
& \bar{Q} \\
& G
\end{aligned}
$$

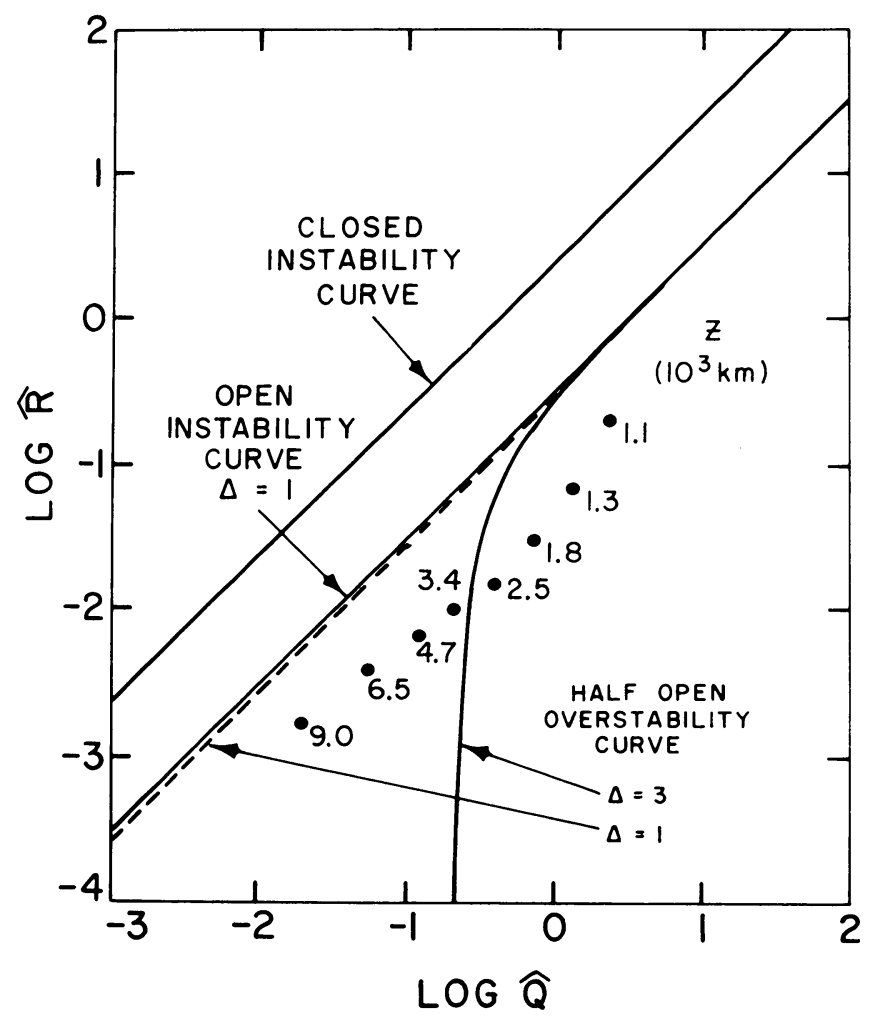

FIG. 6. $\hat{R}, \hat{Q}$ stability diagram including standing gravity waves obtained by Savage (1967). The solid curves are open instability and overstability curves for $\Delta=\rho_{\mathrm{down}} / \rho_{\mathrm{up}}=1.1,3$ and 10 and for $G=69.5$ and $s=1$. The upper short-dashed curve is the closed instability curve. The two long dashed curves are open $(\Delta=1)$ instability and overstability curves. This stability plot is valid for the surface layer of a sunspot.

where $G$, a dimensionless gravity, is given by

$$
G=\begin{gathered}
g d^{3} \\
\kappa^{2} \pi^{3}
\end{gathered}
$$


The $\hat{R}, \hat{Q}$ coordinates eliminate the $\kappa^{2}$ dependence of both $\bar{R}$ and $\bar{Q}$ and emphasize the effect of gravity waves.

It is evident from Figure 6 (which is based on a typical value of $G$ for the surface layer of a sunspot) that the gravity waves produce a large change in the open overstability curve for $\hat{Q}<1$. The physical reason for this large change can be seen with the aid of Figure 7, which shows the fraction of the energy of an incident Alfvén wave

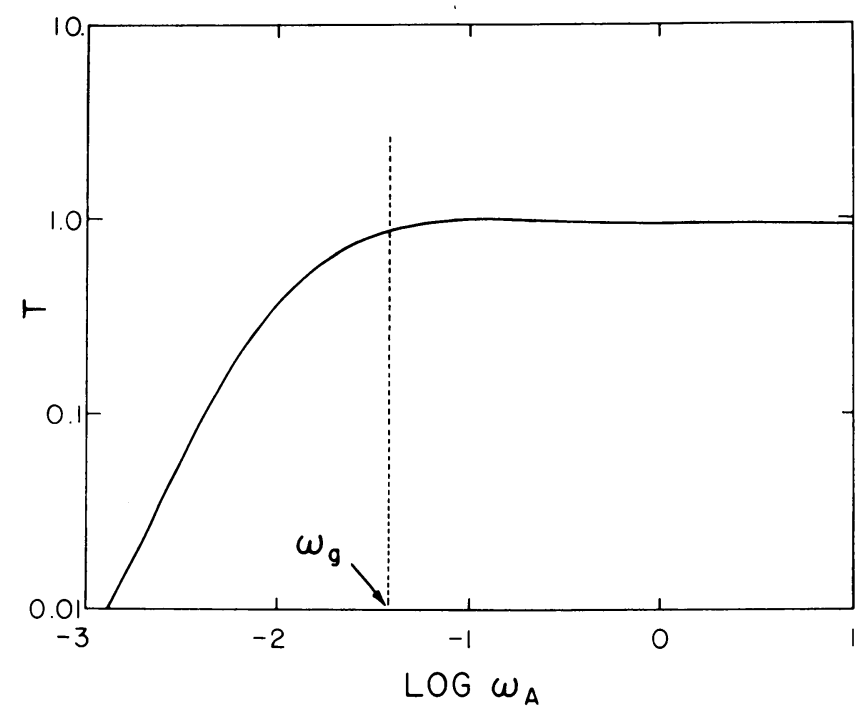

FIG. 7. The energy flux transmission coefficient of Alfvén waves through a density interface as a function of $\left(\omega_{\mathrm{A}}\right.$, the Alfven wave frequency. We assume the horizontal wave number, $k_{\mathrm{H}}$ is $10^{-7} \mathrm{~cm}^{-1}$, $V_{\mathrm{A}}=10^{6} \mathrm{~cm} / \mathrm{sec}$ (the incident Alfvén velocity) and $\Delta=3$. The vertical dashed line indicates the gravity wave frequency.

which is transmitted by the interface. For frequencies larger than $\omega_{\mathrm{g}}$, the frequency of the gravity wave oscillations, the transmission is nearly unity. The slight difference from unity is a result of a small amount of wave reflection due to the change in the Alfvén velocity at the interface. For frequencies less than $\omega_{\mathbf{g}}$, however, the amount of transmitted energy is very much smaller. For these frequencies, the gravity waves respond to perturbations at the interface caused by the incident Alfvén wave in a time which is short compared with the period of the Alfvén wave. This has the result that the reflection coefficient increases greatly and correspondingly produces a large decrease in the overstability curve. The reason that gravity waves affect the overstability curves for $\hat{Q}<1$ can be understood in terms of the above discussion by noting that $\hat{Q}$ can be written as

$$
\hat{Q}=\left(\begin{array}{c}
V_{\mathrm{A}} \\
V_{\mathrm{g}}
\end{array}\right)^{2}
$$


where $V_{\mathrm{A}}$ is the Alfvén velocity and where $V_{\mathrm{g}}$ is the velocity of gravity waves having a wavelength equal to twice the depth of the superadiabatic layer.

Thus for $\hat{Q}<1$, the gravity waves reduce the energy loss by hydromagnetic wave emission and therefore tend to 'close' the layer. This 'closing' can also be seen in the

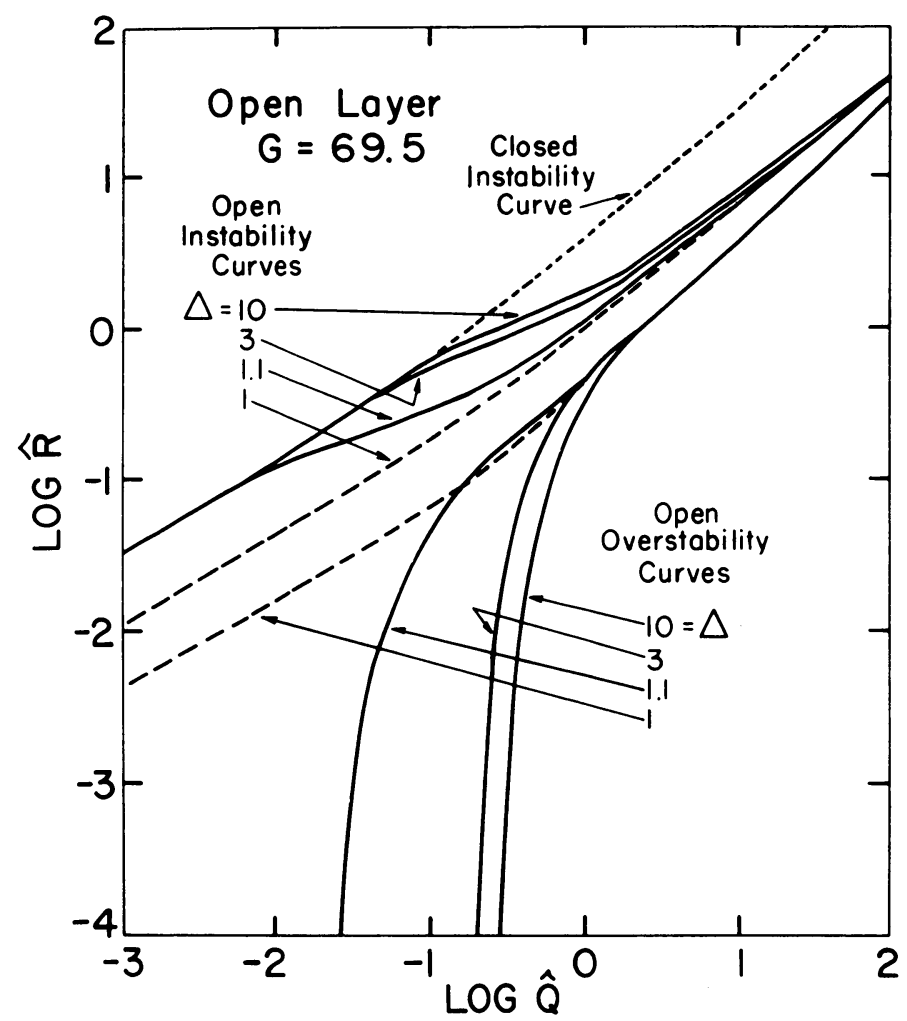

FIG. 8. An estimated $\hat{R}, \hat{Q}$ stability diagram (including gravity waves) which is valid for deep umbral layers $\left(G \approx 10^{13}\right)$. The $\hat{R}, Q$ coordinates of Yun's (1967) sunspot model are included.

instability curves shown in Figure 6 where the transition from the open instability curve for $\hat{Q}>1$ to the closed instability curve for $\hat{Q}<1$ is evident.

Figure 8 shows an estimated $\hat{R}, \hat{Q}$ diagram valid for deep umbral layers $\left(G \approx 10^{13}\right)$. The open overstability curve is for $\Delta=3$, which may be the most appropriate curve for our purposes (Savage, 1967). The $\hat{R}, \hat{Q}$ coordinates for each pressure scale height in the model computed by Yun (1967) are also shown. One sees that for $Z \gtrsim 3000 \mathrm{~km}$ (about $2000 \mathrm{~km}$ below the surface of the sunspot) one is in a regime of overstable oscillations. And if the characteristic depth $d$ for this stability theory is more than one 
pressure scale height (as may well be the case in a vertical magnetic field) the upper three scale heights may also lie in the overstable region.

It therefore seems that overstable oscillatory modes can occur in the interior of sunspots but that unstable convective modes do not occur. However, one should not forget that the existence of the overstable modes is based on a rather crude hydromagnetic model which, strictly speaking, should only be applied to the surface layer of the sunspot. The situation may alter a great deal when the stability of more realistic sunspot models are investigated. With these reservations, some possible consequences of overstable oscillations are discussed in the next section.

\section{Some Possible Consequences of Oscillatory Modes in Sunspots}

If oscillations occur in the interior of sunspots, one can compute some characteristic periods from the hydromagnetic velocity by choosing the vertical wavelength to be equal to $2 d$, where $d$ is the characteristic depth in the stability calculations. This corresponds to nearly closed boundaries for the layer under consideration. And if we choose $d$ to be the pressure scale height, we find (as shown in Figure 9) that the predicted period of oscillation varies from about $1 \mathrm{~min}$ at the surface of the sunspot

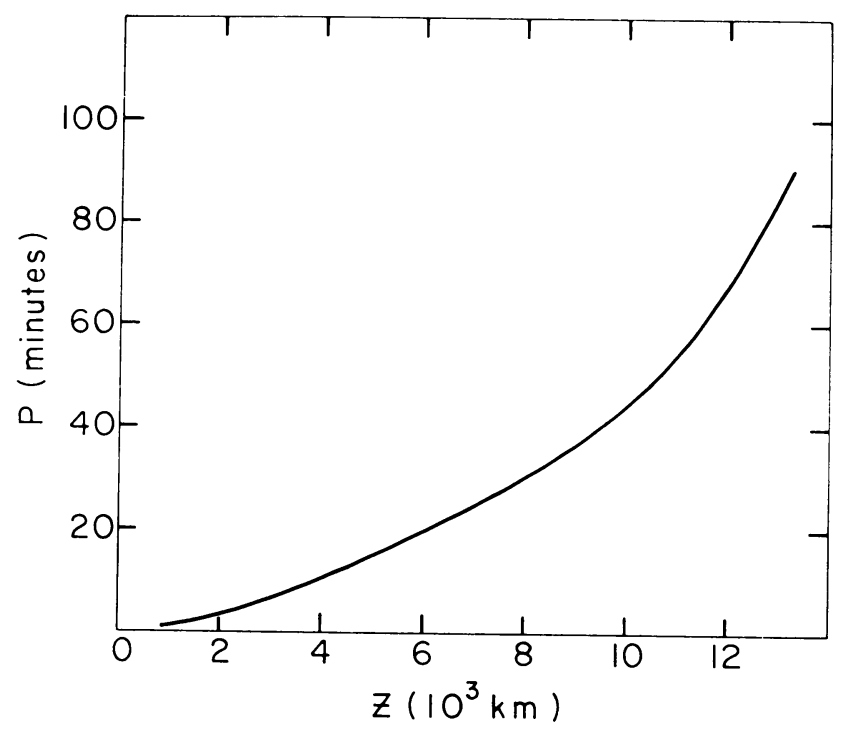

FIG. 9. Period of oscillation (in minutes) of umbral layers as a function of depth into the sunspot.

$\left(Z \approx 1 \times 10^{3} \mathrm{~km}\right)$ to nearly 1 hour at a depth of $10^{4} \mathrm{~km}\left(Z \approx 11 \times 10^{3} \mathrm{~km}\right)$ below the surface. The latter depth is about equal to the umbral diameter for a sunspot having a total area of about $150 \times 10^{-6}$ of the solar hemisphere. 
Thus at depths of $5 \times 10^{3}$ to $10^{4} \mathrm{~km}$ beneath the surface of the sunspot, one expects periods of the order of $\frac{1}{2}$ hour. It is interesting to note that the lifetime of the umbra dots is also about $\frac{1}{2}$ hour. This suggests the possibility that the umbral dots are caused by some sort of 'overshoot' from overstable oscillations deep in the interior of the sunspot.

Another possible consequence of oscillations in the interior of a sunspot is that they may play an important role in the missing energy of sunspots. Some of the missing energy is undoubtedly radiated upward at the surface of the sunspot in the form of hydromagnetic waves. Indeed if the observed 'turbulent velocities' in sunspots are interpreted as displacement velocities in a hydromagnetic traveling wave, the resulting non-thermal flux will be

$$
F=\frac{1}{2} \rho V_{\max }^{2} V_{\mathrm{A}}
$$

and if we choose the density $(\rho)$ at which the maximum turbulent velocities $\left(V_{\max }\right)$ are measured to be approximately $5 \times 10^{-7} \mathrm{gm} / \mathrm{cm}^{3}$, we find that (for $H=2500$ gauss) the Alfvén velocity $\left(V_{\mathrm{A}}\right)$ is approximately $10^{6} \mathrm{~cm} / \mathrm{sec}$. Then for $V_{\max }=3 \times 10^{5} \mathrm{~cm} / \mathrm{sec}$ (r.m.s. turbulent velocity $=2 \times 10^{5} \mathrm{~cm} / \mathrm{sec}$ ), we find that $F=2 \times 10^{10} \mathrm{ergs} / \mathrm{cm}^{2} \mathrm{sec}$ which is a significant fraction of the solar flux in the undisturbed photosphere (i.e. $6 \times 10^{10} \mathrm{ergs} / \mathrm{cm}^{2} \mathrm{sec}$ ).

The above flux is essentially the maximum possible mechanical flux that can be radiated from the surface of the umbra, but it is still a factor of 2.5 less than the missing energy in the umbra. The latter quantity is about $5 \times 10^{10} \mathrm{ergs} / \mathrm{cm}^{2} \mathrm{sec}$ based on an effective temperature of $3700^{\circ} \mathrm{K}$ and it is far from certain that the observed turbulent velocities have the proper phase relation with height to represent an upward traveling wave. Indeed, a flux of $2 \times 10^{10} \mathrm{ergs} / \mathrm{cm}^{2} \mathrm{sec}$ emitted by an umbra having a diameter of $10^{4} \mathrm{~km}$ amounts to nearly $2 \times 10^{28} \mathrm{ergs} / \mathrm{sec}$. This amount of energy is sufficient to keep a small flare going continuously! Thus while hydromagnetic wave emission by the surface of the umbra may account for some of the missing energy, it does not seem possible to account for it all by this means.

Oscillations in the interior of sunspots suggest another possible way for accounting for some of the missing energy. It seems likely that the oscillations in the sunspot would give rise to horizontally propagating hydromagnetic waves (i.e. longitudinal magnetosonic waves). Since the speed of sound is several times larger than the Alfvén velocity at depths of 5000 to $10000 \mathrm{~km}$ in the spot, the longitudinal magnetosonic wave velocity inside the sunspot is approximately the same as the sound velocity outside the spot. At these depths, the speed of sound is of the order of $20 \mathrm{~km} / \mathrm{sec}$ and the density is of the order of $5 \times 10^{-5} \mathrm{gm} / \mathrm{cm}^{2}$. One may estimate the horizontal flux from an equation similar to Equation (9) and one finds the horizontal fluxes equal to $5 \times 10^{11} \mathrm{ergs} / \mathrm{cm}^{2} \mathrm{sec}$ (nearly 10 times the normal photospheric flux) for displacement velocities of $1 \mathrm{~km} / \mathrm{sec}$. And for this flux, the total energy emitted by a cylindrical surface having a diameter of $10^{4} \mathrm{~km}$ and a depth of $5 \times 10^{3} \mathrm{~km}$ is nearly $10^{30} \mathrm{ergs} / \mathrm{sec}$. 
This is approximately equal to the missing energy of an entire sunspot having a penumbral diameter of $2.5 \times 10^{4} \mathrm{~km}$ (and an umbral diameter of $10^{4} \mathrm{~km}$ ) if one takes $\frac{1}{3}$ of the photospheric flux over the entire sunspot area to be the missing energy.

The numbers in the above paragraph are very speculative, but they may indicate one way to account for the missing energy in sunspots. And if this be the case, the lack of a substantial bright ring near sunspots could be understood if the horizontally propagating waves travel several times the penumbral radius of the sunspot before dissipating. Also some energy may be propagated away from the sunspot by means of surface gravity waves.

A third implication of oscillatory modes in the sunspot interior is that they may lend some support to the use of a reduced mixing length by Deinzer (1965) and Yun (1967) in computing models of the sunspot interior. In the absence of a magnetic field, the reason for using a mixing length (equal to the local-pressure scale height, for example) is that an upward-moving element goes roughly one scale height before stopping and giving up its excess thermal energy to its surroundings. In the case of oscillations in the presence of a vertical magnetic field, however, the upward-moving elements do not stop and mix with the surroundings, but reverse their motion instead. Thus the moving elements have a limited amount of time in which to exchange energy with respect to their surroundings and therefore the use of a mixing length which is only a fraction of a scale height seems like a plausible first approximation to estimating the non-radiative energy transport in a sunspot.

In the event that horizontally propagating hydromagnetic waves are emitted in the interior of the sunspot, a modification to the procedure used by Deinzer (1965) and by Yun (1967) is suggested. Namely, the total flux is not constant in the sunspot but decreases with height as energy is radiated horizontally. Thus, the flux should be varied with the depth in future models. One consequence of this is that the larger flux in the deeper portions of a sunspot would produce a larger superadiabatic gradient and, therefore, a greater instability to oscillations.

\section{Appendix}

In mixing length theory, the convective flux $F_{\mathrm{c}}$ is given by (Böhm-Vitense, 1958)

$$
F_{\mathrm{c}}=\frac{1}{2} C_{p} \rho T V\left(\begin{array}{l}
l \\
h
\end{array}\right)\left(\nabla-\nabla^{\prime}\right),
$$

where $C_{p}$ is the heat capacity per gram, $\rho$ is the density, $T$ is the temperature, $V$ is the mean velocity of a 'turbulent element', $l$ is Prandtl's mixing length, $h$ is the pressure scale height, $\nabla=(\mathrm{d}(\ln T)) /(\mathrm{d}(\ln P))$, where $p$ is the gas pressure, and where $\nabla^{\prime}$ is the same quantity in a 'turbulent element'. Similarly (Böhm-Vitense, 1958),

$$
V^{2}={ }_{8 M}^{R T} Q\left(\begin{array}{l}
l \\
h
\end{array}\right)^{2}\left(\nabla-\nabla^{\prime}\right)
$$


where $R$ is the gas constant, where $M$ is the mean molecular weight, and where the parameter $Q=1-(\partial \ln M) /(\partial \ln T)$.

Eliminating $\left(\nabla-\nabla^{\prime}\right)$ from the above two equations and defining the equipartition magnetic field $H_{\text {eq }}$ by

one obtains

$$
\begin{gathered}
\mu H_{\mathrm{eq}}^{2} \\
8 \pi
\end{gathered}=\frac{1}{2} \rho V^{2}
$$

$$
H_{\mathrm{eq}}=\left(\frac{4 \pi}{\mu}\right)^{1 / 2} \rho^{1 / 6} Q^{1 / 3}\left(\frac{F_{\mathrm{c}}}{4}\right)^{1 / 3}\left(\frac{R}{M C_{p}}\right)^{1 / 3}\left(\begin{array}{l}
l \\
h
\end{array}\right)^{1 / 3},
$$

where $\mu$ is the magnetic permeability.

Since $F_{\mathrm{C}} \leqslant F$, where $F$ is the total flux, and since $R / M C_{p} \leqslant 0.4$ (the value for a monatomic gas), one obtains

$$
H_{\mathrm{eq}} \leqslant 6600 \text { gauss }\left(\rho\left[\frac{\mathrm{gm}}{\mathrm{cm}^{3}}\right]\right)^{1 / 6}\left(\begin{array}{l}
l \\
h
\end{array}\right)^{1 / 3} Q^{1 / 3} .
$$

The parameter $Q$ differs from unity only in the upper few thousand kilometers of the normal convection zone (where it takes on values of the order of 2). Therefore $Q$ may be set equal to unity for the purposes of this paper and

$$
H_{\text {eq }} \leqslant 6600 \text { gauss }\left(\rho\left[\frac{\mathrm{gm}}{\mathrm{cm}^{3}}\right]\right)^{1 / 6}\left(\begin{array}{l}
l \\
h
\end{array}\right)^{1 / 3} .
$$

\section{Acknowledgements}

We wish to express our indebtedness to Mr.H.S. Yun of the University of Indiana for the use of his sunspot models prior to publication.

This paper is part of Project Stratoscope of Princeton University sponsored by NSF, ONR, and NASA.

\section{References}

Beckers, J., Schröter, E. (1967) private communication.

Böhm, K.H. (1963) Astrophys. J., 137, 881.

Böhm-Vitense, E. (1958) Z. Astrophys., 46, 108.

Bray, R., Loughhead, R. (1959) Austr. J. Phys., 12, 320.

Brückner, G. (1965) in I.A.U. Symposium No. 22: Solar and Stellar Magnetic Fields, Ed. by R. Lüst, North-Holland Publ. Co., Amsterdam, p. 293.

Chandrasekhar, S. (1961) Hydrodynamic and Hydromagnetic Stability, Oxford University Press, London.

Chitre, S. (1963) Mon. Not. R. astr. Soc., 126, 431.

Danielson, R.E. (1961) Astrophys. J., 134, 289.

Danielson, R.E. (1964) Astrophys. J., 139, 45.

Danielson, R.E. (1965) in I.A.U. Symposium No. 22: Solar and Stellar Magnetic Fields, Ed.

by R. Lüst, North-Holland Publ. Co., Amsterdam, p. 315.

Danielson, R.E. (1966) in Convegno Sulle Machie Solare, Ed. by Barbera (Firenze). 
Deinzer, W. (1965) Astrophys. J., 141, 548.

Elsässer, H., Fricke, K. (1965) in I.A.U. Symposium No. 22: Solar and Stellar Magnetic Fields,

Ed. by R. Lüst, North-Holland Publ. Co., Amsterdam, p. 297.

Elste, G. (1963) J. Quant. Spect. Rad. Trans., 3, 185.

Howard, R. (1958) Astrophys. J., 127, 108.

Kato, S. (1966) P.A.S.J., 18, 201.

Marik, M. (1966) Soviet Astr. - A.J., 10, 315.

Musman, S. (1967) Astrophys. J., 149, 201.

Savage, B.D. (1967) Ph.D. Thesis, Princeton University.

Spiegel, E. A., Veronis, G. (1960) Astrophys. J., 131, 442.

Vitense, E. (1953) Z. Astrophys., 32, 135.

Weiss, N.O. (1964) Proc. Roy. Soc., A256, 99.

Yun, H.S. (1967) private communication.

\section{DISCUSSION}

Severny: Should there exist some phase shift in oscillations at different levels inside a sunspot?

Danielson: Yes, oscillations at different levels inside the sunspot will be coupled to some extent since they are traversed by the same magnetic field. However, the extent of this coupling will not be known until the actual modes in Deinzer's model are computed.

Maltby: Since you have mentioned the transport of energy by sound waves in the horizontal direction, I would like to draw attention to a paper by G. Eriksen and myself (to be published in Solar Physics) where the Evershed effect is explained as a wave phenomenon and the effect of energy transport is mentioned.

Sturrock: Sunspots normally begin as a small dark pore, giving the impression that, when the magnetic-field strength grows to a certain value, the normal convection pattern is suppressed and some other pattern is set up. Can your theory predict the field strength at which a pore would form?

Danielson: Our stability model predicts that the normal convection pattern begins to be suppressed at the instability curve. The magnetic field corresponding to the half open instability curve is of the order of $\mathbf{8 0 0}$ gauss, but this value should not be taken too seriously since our stability model is rather crude.

Elske Smith: Some very tentative results of my study of vertical velocities in the umbra of sunspots may have some bearing on Danielson's model. I have found downward velocities, relative to the photosphere, of the order of $0.3 \mathrm{~km} / \mathrm{sec}$ in certain photospheric lines $(\lambda 5123,5434$, and 5576). There is an indication that these velocities show a time variation with a period of about $5 \mathrm{~min}$. Hence the actual velocities range from almost zero to $0.6 \mathrm{~km} / \mathrm{sec}$ or even higher, though almost always downward. Weaker lines, like $\lambda 5436$ ( $\mathrm{Ni}$ I), arising from greater depths in the umbra, have smaller velocities, on the average of the order of $0.1 \mathrm{~km} / \mathrm{sec}$. 